\title{
Combinatorics of Matrix Factorizations and Integrable Systems
}

Anton Dzhamay

To cite this article: Anton Dzhamay (2013) Combinatorics of Matrix Factorizations and Integrable Systems, Journal of Nonlinear Mathematical Physics 20: Supplement 1, 3447, DOI: https://doi.org/10.1080/14029251.2013.862433

To link to this article: https://doi.org/10.1080/14029251.2013.862433

Published online: 04 January 2021 


\title{
Combinatorics of Matrix Factorizations and Integrable Systems
}

\author{
Anton Dzhamay \\ School of Mathematical Sciences, The University of Northern Colorado, Campus Box 122, \\ 501 20th Street, Greeley, CO 80639, USA \\ adzham@unco.edu \\ Received 2 september 2012 \\ Accepted 13 June 2013
}

\begin{abstract}
We study relations between the eigenvectors of rational matrix functions on the Riemann sphere. Our main result is that for a subclass of functions that are products of two elementary blocks it is possible to represent these relations in a combinatorial-geometric way using a diagram of a cube. In this representation, vertices of the cube represent eigenvectors, edges are labeled by differences of locations of zeroes and poles of the determinant of our matrix function, and each face corresponds to a particular choice of a coordinate system on the space of such functions. Moreover, for each face this labeling encodes, in a neat and efficient way, a generating function for the expressions of the remaining four eigenvectors that label the opposing face of the cube in terms of the coordinates represented by the chosen face. The main motivation behind this work is that when our matrix is a Lax matrix of a discrete integrable system, such generating functions can be interpreted as Lagrangians of the system, and a choice of a particular face corresponds to a choice of the direction of the motion.
\end{abstract}

Keywords: discrete integrable systems; matrix refactorization; discrete Painlevé equations.

2000 Mathematics Subject Classification: 37K32, 34M56, 39A10, 37K20

\section{Introductions}

Over the last 25 years a lot of research efforts have been directed towards the study of discrete analogues of integrable systems and, in particular, on how to adapt the existing methods and techniques from the classical theory of differential completely integrable systems to the discrete case. The present paper is a small part of a larger project that aims to understand the Lagrangian structure of discrete integrable systems directly in terms of their Lax matrices and is motivated by work of Krichever and Phong [6], who obtained expressions for a universal symplectic form and elementary generating Hamiltonians on the space of Lax matrices for continuous completely integrable systems. Note that a universal formula for a Lagrangian description of integrable systems in the continuous case was obtained by Zakharov and Mikhailov [14].

For discrete completely integrable systems, the Lagrangian point of view has been developed in the classical papers by Veselov [12,13] and Moser and Veselov [8]. Recently a very promising approach to the study of Lagrangian structure of integrable lattice equations in terms of Lagrangian multiforms has been proposed by Nijhoff and Lobb [7]. This approach is related to the notion of multidimensional consistency formulated by Bobenko and Suris [2] and independently by Nijhoff [9]. Although our approach is much more elementary and is based on the notion of the refactorization transformations, as in the original work of Veselov and Moser, the resulting combinatorial diagrams encoding such transformations are reminiscent of the multidimensional consistency approach, and it will be very interesting to see if this is more than a pure coincidence.

The appearance of refactorization transformations in the theory of discrete integrable systems is not very surprising. Indeed, a discrete analogue of the Lax Pair representation is the isospectral 
transformation $\tilde{\mathbf{L}}(z)=\mathbf{R}(z) \mathbf{L}(z) \mathbf{R}(z)^{-1}$, where $\mathbf{L}(z)$ is the Lax matrix of the system and $\mathbf{R}(z)$ is the evolution matrix that has to be chosen in a special way dependent on $\mathbf{L}(z)$. When $\mathbf{L}(z)$ is a rational matrix function with the fixed singularity structure, one can specify elementary evolution matrices $\mathbf{R}(z)$ using pairs of points $p_{ \pm}$on the spectral curve $\Gamma$ of $\mathbf{L}(z)$ and the eigenvectors of $\mathbf{L}(z)$ at those points, and choosing those points to lie above the points of the determinantal divisor of $\operatorname{det} \mathbf{L}(z)$ corresponds to factoring $\mathbf{L}(z)=\mathbf{L}_{1}(z) \mathbf{B}_{2}(z)$ and mapping it to $\tilde{\mathbf{L}}(z)=\mathbf{B}_{2}(z) \mathbf{L}_{1}(z)$, i.e., by choosing $\mathbf{R}(z)=\mathbf{B}_{2}(z)$.

In describing such refactorization transformations it is essential to choose a good coordinate system on the space of Lax matrices. The natural candidates for the coordinates are the eigenvectors of $\mathbf{L}(z)$ and $\mathbf{L}(z)^{-1}$. However, for the refactorization transformations another important class of vectors consists of vectors defining elementary building blocks. The relationship between these two sets of vectors is quite complicated, and in an attempt to understand it we noticed a very elegant way to represent this relationship using geometric diagrams described in the present paper. Although we restrict our attention to the quadratic case when $\mathbf{L}(z)$ is a product of two blocks and when there is no essential difference between the vectors of the additive and multiplicative representation, it is still quite interesting. Indeed, such refactorization transformations describe, for example, the change of polarization in the interaction of soliton solutions of the matrix $\mathrm{KdV}$ equations [5] and integrable discrete vector nonlinear Schrödinger equations [10]. Further, in [4] we showed that for two-dimensional matrix functions the directional dynamics of the eigenvectors is described by difference Painlevé-V equation.

We plan to use the tools developed in this paper to study the higher dimensional case which considers products of three and more elementary blocks and, in particular, the relation to YangBaxter maps, in a separate publication.

This paper is organized as follows. In the next section we briefly describe a particular class of Lax matrices that we consider, their additive and multiplicative representations, and the notion of an elementary divisor - a building block for a multiplicative representation. In Section 3 we explain a visual representation of some linear equations involving elementary divisors and in Section 4 we use this visual representation to describe the relations between eigenvectors of a quadratic Lax matrix. We also show how to use the resulting cube diagram to generate the Lagrangian functions of the refactorization transformations.

\section{Preliminaries}

The main goal of this section is to describe the space of Lax matrices, their additive and multiplicative representations and related coordinate systems, and briefly review the relationship between isomonodromic transformations and discrete Painlevé equations, see [4] for details.

\subsection{The space of Lax Matrices}

We consider the space $\mathscr{L}$ of rational $m \times m$ matrix functions $\mathbf{L}(z)$ satisfying the following conditions. We assume that $\mathbf{L}(z)$ is regular, diagonalizable (and diagonalized) at some normalization point $z_{0}$ that we take to be $z_{0}=\infty$,

$$
\mathbf{L}_{0}=\lim _{z \rightarrow \infty} \mathbf{L}(z)=\operatorname{diag}\left\{\rho_{1}, \ldots, \rho_{m}\right\} .
$$

We also assume that the singularity structure of $\mathbf{L}(z)$, i.e., points where $\mathbf{L}(z)$ has a pole or becomes degenerate, is accurately represented by its determinant, and that the determinant is generic, i.e., it 
has only simple zeroes and simple poles. This amounts to requiring that $\mathbf{L}(z)$ is holomorphic except for simple poles at the points $z_{1}, \ldots, z_{k}, \mathbf{M}(z)=\mathbf{L}(z)^{-1}$ is holomorphic except for simple poles at the points $\zeta_{1}, \ldots, \zeta_{k}$, all $z_{i}$ and $\zeta_{j}$ are distinct, and

$$
\operatorname{det} \mathbf{L}(z)=\rho_{1} \cdots \rho_{m} \frac{\prod_{\alpha}\left(z-\zeta_{\alpha}\right)}{\prod_{k}\left(z-z_{k}\right)} .
$$

These conditions mean that the residues $\mathbf{L}_{i}=\operatorname{res}_{z_{i}} \mathbf{L}(z)$ and $\mathbf{M}_{j}=-\operatorname{res}_{\zeta_{j}} \mathbf{M}(z)$, where the negative sign here is just for convenience, are matrices of rank one. Using the $\uparrow$ symbol to indicate a row vector, we have:

$$
\begin{aligned}
\mathbf{L}(z) & =\mathbf{L}_{0}+\sum_{i=1}^{k} \frac{\mathbf{L}_{i}}{z-z_{i}}, \quad \text { where } \mathbf{L}_{0}=\operatorname{diag}\left\{\rho_{1}, \ldots, \rho_{m}\right\} \text { and } \mathbf{L}_{i}=\mathbf{a}_{i} \mathbf{b}_{i}^{\dagger}, \\
\operatorname{det} \mathbf{L}(z) & =\rho_{1} \cdots \rho_{m} \frac{\prod_{i=1}^{k}\left(z-\zeta_{i}\right)}{\prod_{j=1}^{k}\left(z-z_{j}\right)} \\
\mathbf{L}(z)^{-1}=\mathbf{M}(z) & =\mathbf{M}_{0}-\sum_{i=j}^{k} \frac{\mathbf{M}_{j}}{z-\zeta_{j}}, \quad \text { where } \mathbf{M}_{0}=\mathbf{L}_{0}^{-1}, \quad \mathbf{M}_{j}=\mathbf{c}_{j} \mathbf{d}_{j}^{\dagger},
\end{aligned}
$$

The above representations of $\mathbf{L}(z)$ and $\mathbf{M}(z)$ are called additive representations and the vectors $\mathbf{a}_{i}$, $\mathbf{b}_{i}^{\dagger}$ (resp. $\mathbf{c}_{i}, \mathbf{d}_{i}^{\dagger}$ ) are called additive eigenvectors of $\mathbf{L}(z)$ (resp. $\mathbf{M}(z)$ ). Note that these eigenvectors are also characterized by $\mathbf{L}\left(\zeta_{\alpha}\right) \mathbf{c}_{\alpha}=\mathbf{M}\left(z_{i}\right) \mathbf{a}_{i}=\mathbf{0}$ and $\mathbf{d}_{\alpha}^{\dagger} \mathbf{L}\left(\zeta_{\alpha}\right)=\mathbf{b}_{i}^{\dagger} \mathbf{M}\left(z_{i}\right)=\mathbf{0}$.

Let $\mathscr{D}=\sum_{i} z_{i}-\sum_{i} \zeta_{i}$ be the divisor of the (determinant) of $\mathbf{L}(z)$ and denote the space of matrices $\mathbf{L}(z)$ satisfying conditions (2.3)-(2.5) by $\mathscr{M}_{r}^{\mathscr{D}}$. Then eigenvectors give coordinates on this space, as described by the following lemma.

Lemma 2.1. Generically, the collection $\left\{\mathbf{a}_{k}, \mathbf{d}_{k}^{\dagger}\right\}_{k=1}^{n}$ (or the collection $\left\{\mathbf{c}_{k}, \mathbf{b}_{k}^{\dagger}\right\}_{k=1}^{n}$ ) is a coordinate system on the space $\mathscr{M}_{r}^{\mathscr{D}}$.

Proof. Consider the equations $\mathbf{M}\left(z_{k}\right) \mathbf{a}_{k}=\mathbf{0}$ and $\mathbf{d}_{i}^{\dagger} \mathbf{L}\left(\zeta_{i}\right)=\mathbf{0}$ :

$$
\mathbf{L}_{0}^{-1} \mathbf{a}_{k}-\sum_{i=1}^{n} \mathbf{c}_{i} \frac{\mathbf{d}_{i}^{\dagger} \mathbf{a}_{k}}{z_{k}-\zeta_{i}}=\mathbf{0}, \quad \mathbf{d}_{i}^{\dagger} \mathbf{L}_{0}+\sum_{k=1}^{n} \frac{\mathbf{d}_{i}^{\dagger} \mathbf{a}_{k}}{\zeta_{i}-z_{k}} \mathbf{b}_{k}^{\dagger}=\mathbf{0} .
$$

Then if the matrix $\left[\frac{\mathbf{d}_{\mathbf{i}}^{\dagger} \mathbf{a}_{k}}{z_{k}-\zeta_{i}}\right]$ is invertible,

$$
\mathbf{c}_{i}=\mathbf{L}_{0}^{-1} \mathbf{a}_{k}\left[\frac{\mathbf{d}_{i}^{\dagger} \mathbf{a}_{k}}{z_{k}-\zeta_{i}}\right]^{-1}, \quad \mathbf{b}_{k}^{\dagger}=\left[\frac{\mathbf{d}_{i}^{\dagger} \mathbf{a}_{k}}{z_{k}-\zeta_{i}}\right]^{-1} \mathbf{d}_{i}^{\dagger} \mathbf{L}_{0} .
$$

\subsection{Elementary Divisors and Multiplicative Representations}

To define a multiplicative representation of $\mathbf{L}(z)$ we first define its building blocks. These are rational matrix functions of the special form

$$
\mathbf{B}(z)=\mathbf{I}+\frac{\mathbf{G}}{z-z_{0}}, \quad \text { where } \mathbf{G}=\mathbf{f g}^{\dagger} \text { is a matrix of rank } 1 .
$$


We call such matrices elementary divisors [4]. If $\operatorname{tr}(\mathbf{G})=\mathbf{g}^{\dagger} \mathbf{f} \neq 0$, we can consider instead of $\mathbf{G}$ a rank-one projector $\mathbf{P}=\mathbf{f}\left(\mathbf{g}^{\dagger} \mathbf{f}\right)^{-1} \mathbf{g}^{\dagger}$. Then we can write

$$
\mathbf{B}(z)=\mathbf{B}\left(z ; z_{0}, \zeta_{0}\right)=\mathbf{I}+\frac{z_{0}-\zeta_{0}}{z-z_{0}} \frac{\mathbf{f g}^{\dagger}}{\mathbf{g}^{\dagger} \mathbf{f}}, \quad \operatorname{det} \mathbf{B}(z)=\frac{z-\zeta_{0}}{z-z_{0}}, \quad \mathbf{B}(z)^{-1}=\mathbf{I}+\frac{\zeta_{0}-z_{0}}{z-\zeta_{0}} \frac{\mathbf{f g}^{\dagger}}{\mathbf{g}^{\dagger} \mathbf{f}} .
$$

If we now pair the zeroes and poles of $\operatorname{det} \mathbf{L}(z)$ in some way as $\left(\zeta_{s}, z_{s}\right)$, then, for any such pair, there is a multiplicative component of $\mathbf{L}(z)$ of the above form. There are two ways to think about such components — we can look at factors or at divisors, see also [1].

Definition 2.1. We say that elementary divisors $\mathbf{B}_{s}^{r}(z)\left(\operatorname{resp} . \mathbf{B}_{s}^{l}(z)\right)$ corresponding to pairs $\left(\zeta_{s}, z_{s}\right)$ are right (resp. left) divisors of $\mathbf{L}(z)$ if $\mathbf{L}(z)=\mathbf{L}_{s}^{r}(z) \mathbf{B}_{s}^{r}(z)\left(\operatorname{resp} . \mathbf{L}(z)=\mathbf{B}_{s}^{l}(z) \mathbf{L}_{s}^{l}(z)\right)$ where $\mathbf{L}_{s}^{r}(z)$ (resp. $\mathbf{L}_{s}^{l}(z)$ ) is regular at $z_{s}$. Further, we say that elementary divisors $\mathbf{B}_{s}(z)$ corresponding to pairs $\left(\zeta_{s}, z_{s}\right)$ are the factors of $\mathbf{L}(z)$ if $\mathbf{L}(z)=\mathbf{L}_{0} \mathbf{B}_{1}(z) \cdots \mathbf{B}_{k}(z)$.

In [4] we showed that left and right divisors can be written explicitly in terms of the eigenvectors of $\mathbf{L}(z)$,

$$
\mathbf{B}_{s}^{r}(z)=\mathbf{I}+\frac{z_{s}-\zeta_{s}}{z-z_{s}} \frac{\mathbf{c}_{s} \mathbf{b}_{s}^{\dagger}}{\mathbf{b}_{s}^{\dagger} \mathbf{c}_{s}}, \quad \mathbf{B}_{s}^{l}(z)=\mathbf{I}+\frac{z_{s}-\zeta_{s}}{z-z_{s}} \frac{\mathbf{a}_{s} \mathbf{d}_{s}^{\dagger}}{\mathbf{d}_{s}^{\dagger} \mathbf{a}_{s}}
$$

and so the coordinate systems described in Lemma 2.1 are just parameterizations of Lax matrices by left (resp. right) divisors. When $\mathbf{L}(z)$ has only two factors, which is the quadratic case that we focus on in this paper, there is no essential difference between divisors and factors.

\subsection{Re-factorization transformations and $d-P_{V}$}

We now consider the refactorization transformation in the quadratic case. Let

$$
\mathbf{L}(z)=\mathbf{L}_{0} \mathbf{B}_{1}(z) \mathbf{B}_{2}(z)=\mathbf{B}_{2}^{l}(z) \mathbf{L}_{0} \mathbf{B}_{1}^{r}(z)=\mathbf{B}_{1}^{l}(z) \mathbf{L}_{0} \mathbf{B}_{2}^{r}(z)
$$

Then we can consider either isospectral transformation with $\mathbf{R}(z)=\mathbf{B}_{1}^{r}(z)$,

$$
\mathbf{L}(z)=\mathbf{B}_{2}^{l}(z) \mathbf{L}_{0} \mathbf{B}_{1}^{r}(z) \mapsto \tilde{\mathbf{L}}(z)=\mathbf{B}_{1}^{r}(z) \mathbf{B}_{2}^{l}(z) \mathbf{L}_{0}=\tilde{\mathbf{B}}_{2}^{l}(z) \mathbf{L}_{0} \tilde{\mathbf{B}}_{1}^{r}(z),
$$

or isomonodromic transformations

$$
\mathbf{L}(z)=\mathbf{B}_{2}^{l}(z) \mathbf{L}_{0} \mathbf{B}_{1}^{r}(z) \mapsto \tilde{\mathbf{L}}(z)=\mathbf{B}_{1}^{r}(z+1) \mathbf{B}_{2}^{l}(z) \mathbf{L}_{0}=\tilde{\mathbf{B}}_{2}^{l}(z) \mathbf{L}_{0} \tilde{\mathbf{B}}_{1}^{r}(z) .
$$

When $m=2$, the resulting phase space is two-dimensional and it possible to introduce the socalled spectral coordinates $(\gamma, \pi)$ on the space of Lax matrices. Then, in the isomonodromic case, the spectral coordinates of $\mathbf{L}(z)$ and $\tilde{\mathbf{L}}(z)$ are related by the difference Painlevé V equation in the Sakai's classification [11], see [4]:

$$
\begin{aligned}
\tilde{\gamma}+\gamma & =z_{2}+\zeta_{2}+\frac{\rho_{1}\left(k_{1}-z_{1}+\zeta_{2}\right)}{\pi-\rho_{1}}+\frac{\rho_{2}\left(k_{2}-z_{1}+\zeta_{2}+1\right)}{\pi-\rho_{2}}, \\
\tilde{\pi} \pi & =\rho_{1} \rho_{2} \frac{\left(\tilde{\gamma}-\tilde{z}_{2}\right)\left(\tilde{\gamma}-\tilde{\zeta}_{2}\right)}{\left(\tilde{\gamma}-\tilde{z}_{1}\right)\left(\tilde{\gamma}-\tilde{\zeta}_{1}\right)} .
\end{aligned}
$$

In [3] we proved the following 
Theorem 2.1. The equations of both the isospectral and isomonodromic dynamic can be written in the Lagrangian form with

$$
\begin{aligned}
\mathscr{L}(\mathbf{X}, \mathbf{Y}, t)=\left(z_{2}-z_{1}(t)\right) \log \left(\mathbf{x}_{1}^{\dagger} \mathbf{x}_{2}\right)+\left(z_{1}(t)-\zeta_{2}\right) \log \left(\mathbf{y}_{1}^{\dagger} \mathbf{L}_{0}^{-1} \mathbf{x}_{2}\right) \\
\\
+\left(\zeta_{2}-\zeta_{1}(t)\right) \log \left(\mathbf{y}_{1}^{\dagger} \mathbf{L}_{0}^{-1} \mathbf{y}_{2}\right)+\left(\zeta_{1}(t)-z_{2}\right) \log \left(\mathbf{x}_{1}^{\dagger} \mathbf{y}_{2}\right),
\end{aligned}
$$

where $\mathbf{X}=\left(\mathbf{x}_{1}, \mathbf{x}_{2}^{\dagger}\right)$ and $\mathbf{Y}=\left(\mathbf{y}_{1}, \mathbf{y}_{2}^{\dagger}\right)$, in the isomonodromic case $z_{1}(t)=z_{1}-t, \zeta_{1}(t)=\zeta_{1}-t$, and in the isospectral case $z_{1}(t)=z_{1}, \zeta_{1}(t)=\zeta_{1}$ and $\mathscr{L}(X, Y)$ is time-independent.

Here $\mathbf{x}_{i}^{\dagger}, \mathbf{x}_{j}, \mathbf{y}_{i}^{\dagger}, \mathbf{y}_{j}$ are some eigenvectors of $\mathbf{L}(z)$ and $\tilde{\mathbf{L}}(z)$.

The main goal of the present paper is to give a combinatorial-geometric representation of this generating function.

\section{Linear Equations given by Elementary Divisors and their Geometric Representation}

In studying the relationship between various eigenvectors of $\mathbf{L}(z)$ we mainly work with linear equations of the form $\mathbf{v} \sim \mathbf{B}\left(z^{*}\right) \mathbf{w}$ and $\mathbf{v}^{\dagger} \sim \mathbf{w}^{\dagger} \mathbf{B}\left(z^{*}\right)$, where $\sim$ means that the two vectors are proportional and $\mathbf{B}\left(z^{*} ; z_{0}, \zeta_{0}\right)$ is a matrix of the form (2.8) evaluated at some point $z^{*}$. In this section we give a diagrammatic representation of such equations using the language of elementary triples.

\subsection{Basic Definitions}

In what follows, let $\mathbf{V}$ be an $n$-dimensional complex vector space whose elements are column vectors, $\mathbf{V} \simeq \mathbb{C}^{n}$, and let $\mathbf{V}^{\dagger}$ be its dual-space. We think of elements of $\mathbf{V}^{\dagger}$ as row-vectors and denote its elements as $\mathbf{q}^{\dagger} \in \mathbf{V}^{\dagger} \simeq\left(\mathbb{C}^{n}\right)^{\dagger}$. Our main definition is the following.

Definition 3.1. Let $\lambda_{i} \in \mathbb{P} \mathbf{V}, i=1, \ldots 3$, be one-dimensional linear subspaces of $\mathbf{V}$ and $\mu^{\dagger} \in \mathbb{P} \mathbf{V}^{\dagger}$ be a one-dimensional linear subspace of $\mathbf{V}^{\dagger}$. We say that $\left(\lambda_{1}, \lambda_{2}, \lambda_{3}\right)$ form a $\mu^{\dagger}$-based triple with parameters $\left(\alpha_{1}, \alpha_{2}, \alpha_{3}\right), \alpha_{i} \in \mathbb{C} \backslash\{0\}$, if the following conditions hold:

- $\lambda_{i} \notin \operatorname{ker} \mu^{\dagger}$ for all $i$;

- $\lambda_{i} \neq \lambda_{j}$ for $i \neq j$ but $\lambda_{i} \subset \lambda_{j}+\lambda_{k}$ for all $i, j, k$.

- $\sum_{i=1}^{3} \frac{\alpha_{i}}{\mu^{\dagger} \lambda_{i}} \lambda_{i}=\frac{\alpha_{1}}{\mu^{\dagger} \lambda_{1}} \lambda_{1}+\frac{\alpha_{2}}{\mu^{\dagger} \lambda_{2}} \lambda_{2}+\frac{\alpha_{3}}{\mu^{\dagger} \lambda_{3}} \lambda_{3}=0$.

The last equation above adjusts the "slope" of $\lambda_{i}$ w.r.t. $\lambda_{j}$ and $\lambda_{k}$ and it should be interpreted in terms of spanning vectors. To that end, let $\lambda_{i}=\operatorname{Span}_{\mathbb{C}}\left\{\mathbf{p}_{i}\right\}$ and $\mu^{\dagger}=\operatorname{Span}_{\mathbb{C}}\left\{\mathbf{q}^{\dagger}\right\}$. Then we require that the vectors $\mathbf{p}_{1}, \mathbf{p}_{2}$, and $\mathbf{p}_{3}$ are linearly dependent but pairwise independent, $\mathbf{q}^{\dagger} \mathbf{p}_{i} \neq 0$ for all $i$, and the last equation takes the form

$$
\sum_{i=1}^{3} \frac{\alpha_{i}}{\mathbf{q}^{\dagger} \mathbf{p}_{i}} \mathbf{p}_{i}=\frac{\alpha_{1}}{\mathbf{q}^{\dagger} \mathbf{p}_{1}} \mathbf{p}_{1}+\frac{\alpha_{2}}{\mathbf{q}^{\dagger} \mathbf{p}_{2}} \mathbf{p}_{2}+\frac{\alpha_{3}}{\mathbf{q}^{\dagger} \mathbf{p}_{3}} \mathbf{p}_{3}=\mathbf{0}
$$

Note that this equation is homogeneous and so is independent of the choice of basis vectors. We represent such triples by diagrams of the form 

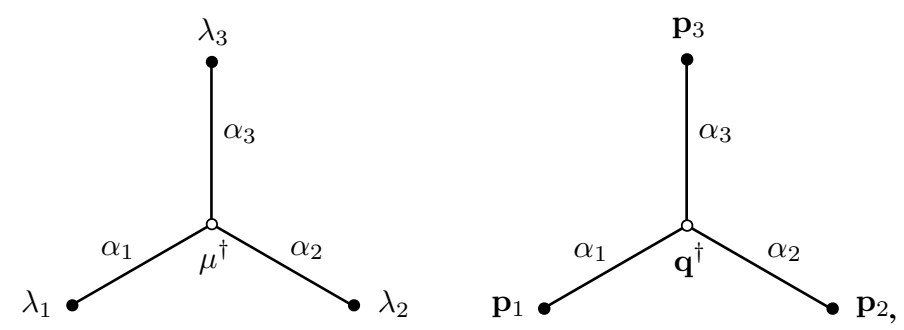

where we use the black circles for subspaces of $\mathbf{V}$ (or their basis vectors) and white circles for subspaces of $\mathbf{V}^{\dagger}$. In what follows, we switch freely between subspace and vector formulations.

It is clear that $\alpha_{i}$ s are defined up to a common multiplicative constant and satisfy

$$
\alpha_{1}+\alpha_{2}+\alpha_{3}=0
$$

Moreover, given $\mathbf{q}^{\dagger}$, there is a unique vector $\left[\mathbf{p}_{i}\right]=\left[\mathbf{p}_{i}\right]_{\mathbf{q}^{\dagger}} \in \lambda_{i}$ normalized by the condition $\mathbf{q}^{\dagger}[\mathbf{p}]=1$, i.e.,

$$
\left[\mathbf{p}_{i}\right]=\frac{\mathbf{p}_{i}}{\mathbf{q}^{\dagger} \mathbf{p}_{i}} \in \lambda_{i},
$$

where $\mathbf{p}_{i}$ is any non-zero vector in $\lambda_{i}$. Then, in terms of the normalized vectors, the linear dependence equation (3.1) becomes

$$
\alpha_{1}\left[\mathbf{p}_{1}\right]+\alpha_{2}\left[\mathbf{p}_{2}\right]+\alpha_{3}\left[\mathbf{p}_{3}\right]=\mathbf{0} \quad \text { or } \quad\left[\mathbf{p}_{i}\right]=\frac{\alpha_{j}\left[\mathbf{p}_{j}\right]+\alpha_{k}\left[\mathbf{p}_{k}\right]}{\alpha_{j}+\alpha_{k}}, \quad i, j, k \text { all distinct },
$$

which explains the interpretation of this equation in terms of "slopes".

Definition 3.2. We define the $\lambda$-based dual triples $\left(\mu_{1}, \mu_{2}, \mu_{3}\right)$ with parameters $\left(\beta_{1}, \beta_{2}, \beta_{3}\right), \beta_{i} \in$ $\mathbb{C} \backslash\{0\}$, where $\lambda \in \mathbb{P V}$ is a one-dimensional linear subspace of $\mathbf{V}$ and $\mu_{i}^{\dagger} \in \mathbb{P} \mathbf{V}^{\dagger}, i=1, \ldots 3$, are onedimensional linear subspaces of $\mathbf{V}^{\dagger}$ in exactly the same way, and represent them by the following diagrams:
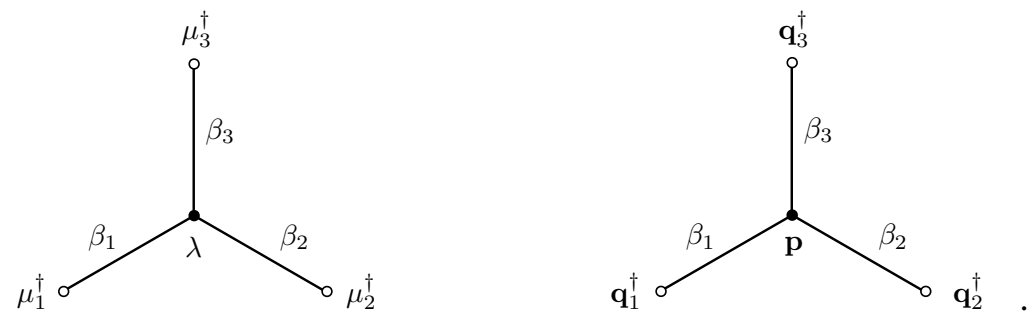

Finally, the behavior of the triples under the action of $\mathbf{G L}(\mathbf{V})$ is described by the following obvious Lemma.

\section{Lemma 3.1. Let $\mathbf{A} \in \mathbf{G L}(\mathbf{V})$.}

(i) If $\left(\lambda_{1}, \lambda_{2}, \lambda_{3}\right)$ is a $\mu^{\dagger}$-based triple with parameters $\left(\alpha_{1}, \alpha_{2}, \alpha_{3}\right)$, then $\left(\mathbf{A} \lambda_{1}, \mathbf{A} \lambda_{2}, \mathbf{A} \lambda_{3}\right)$ is a $\mu^{\dagger} \mathbf{A}^{-1}$-based triple with the same parameters $\left(\alpha_{1}, \alpha_{2}, \alpha_{3}\right)$;

(ii) dually,

$\left(\mu_{1}^{\dagger}, \mu_{2}^{\dagger}, \mu_{3}^{\dagger}\right)$ be a $\lambda$-based triple with parameters $\left(\beta_{1}, \beta_{2}, \beta_{3}\right)$, then $\left(\mu_{1}^{\dagger} \mathbf{A}^{-1}, \mu_{2}^{\dagger} \mathbf{A}^{-1}, \mu_{3}^{\dagger} \mathbf{A}^{-1}\right)$ is an $\mathbf{A} \lambda$-based triple with the same parameters $\left(\beta_{1}, \beta_{2}, \beta_{3}\right)$. 


\subsection{Elementary Divisors and Triples}

We now turn our attention to the study of the relationship between triples and elementary divisors. The following Lemma is immediate.

Lemma 3.2. Let

$$
\mathbf{B}=\mathbf{I}+\frac{z_{0}-\zeta_{0}}{z^{*}-z_{0}} \frac{\mathbf{p q}^{\dagger}}{\mathbf{q}^{\dagger} \mathbf{p}}=\mathbf{I}+\frac{z_{0}-\zeta_{0}}{z^{*}-z_{0}}[\mathbf{p}] \mathbf{q}^{\dagger}=\mathbf{I}+\frac{z_{0}-\zeta_{0}}{z^{*}-z_{0}} \mathbf{p}\left[\mathbf{q}^{\dagger}\right]
$$

$[\mathbf{p}]=[\mathbf{p}]_{\mathbf{q}^{\dagger}}$ and $\left[\mathbf{q}^{\dagger}\right]=\left[\mathbf{q}^{\dagger}\right]_{\mathbf{p}}$. Note that we can normalize either $\mathbf{p}$ or $\mathbf{q}^{\dagger}$, but not both. The choice of which one should be normalized depends on whether we think of $\mathbf{B}$ as acting on the elements of $\mathbf{V}$ or $\mathbf{V}^{\dagger}$. Then

(i) Vectors $\mathbf{p}$ and $\mathbf{q}^{\dagger}$ are the eigenvectors of $\mathbf{B}$,

$$
\mathbf{q}^{\dagger} \mathbf{B}=\frac{z^{*}-\zeta_{0}}{z^{*}-z_{0}} \mathbf{q}^{\dagger}, \quad \mathbf{B} \mathbf{p}=\frac{z^{*}-\zeta_{0}}{z^{*}-z_{0}} \mathbf{p}, \quad \text { where } \quad \operatorname{det} \mathbf{B}=\frac{z^{*}-\zeta_{0}}{z^{*}-z_{0}}
$$

(ii) For any vector $\mathbf{w}$ such that $\mathbf{q}^{\dagger} \mathbf{w} \neq 0$, and for any row-vector $\mathbf{w}^{\dagger}$ such that $\mathbf{w}^{\dagger} \mathbf{p} \neq 0$, using normalized vectors we get

$$
(\mathbf{B}-\mathbf{I})[\mathbf{w}]_{\mathbf{q}^{\dagger}}=\frac{z_{0}-\zeta_{0}}{z^{*}-z_{0}}[\mathbf{p}]_{\mathbf{q}^{\dagger}}, \quad\left[\mathbf{w}^{\dagger}\right]_{\mathbf{p}}(\mathbf{B}-\mathbf{I})=\frac{z_{0}-\zeta_{0}}{z^{*}-z_{0}}\left[\mathbf{q}^{\dagger}\right]_{\mathbf{p}}
$$

(iii) If $\mathbf{v}=\mathbf{B w}$, then $[\mathbf{v}]_{\mathbf{q}^{\dagger}}=\left(z^{*}-z_{0}\right) /\left(z^{*}-\zeta_{0}\right) \mathbf{B}[\mathbf{w}]_{\mathbf{q}^{\dagger}}$, and so $(\mathbf{w}, \mathbf{p}, \mathbf{v})$ form a $\mathbf{q}^{\dagger}$-triple with parameters $\left(z^{*}-z_{0}, z_{0}-\zeta_{0}, \zeta_{0}-z^{*}\right)$. Similarly, if $\mathbf{v}^{\dagger}=\mathbf{w}^{\dagger} \mathbf{B}$, then $\left[\mathbf{v}^{\dagger}\right] \mathbf{p}=\left(z^{*}-z_{0}\right) /\left(z^{*}-\zeta_{0}\right)\left[\mathbf{w}^{\dagger}\right] \mathbf{p} \mathbf{B}$, and $\left(\mathbf{w}^{\dagger}, \mathbf{p}^{\dagger}, \mathbf{v}^{\dagger}\right)$ form a $\mathbf{p}$-triple with parameters $\left(z^{*}-z_{0}, z_{0}-\zeta_{0}, \zeta_{0}-z^{*}\right)$.

(iv) Conversely, for any $\mathbf{q}^{\dagger}$-triple $\left(\mathbf{p}_{1}, \mathbf{p}_{2}, \mathbf{p}_{3}\right)$ with parameters $\left(\alpha_{1}, \alpha_{2}, \alpha_{3}\right)$,

$$
\left[\mathbf{p}_{3}\right]=\frac{\alpha_{1}}{\alpha_{1}+\alpha_{2}}\left(\mathbf{I}+\frac{\alpha_{2}}{\alpha_{1}}\left[\mathbf{p}_{2}\right] \mathbf{q}^{\dagger}\right)\left[\mathbf{p}_{1}\right]=\frac{1}{\alpha_{1}+\alpha_{2}} \frac{\partial}{\partial \mathbf{q}^{\dagger}}\left(\alpha_{1} \log \left(\mathbf{q}^{\dagger} \mathbf{p}_{1}\right)+\alpha_{2} \log \left(\mathbf{q}^{\dagger} \mathbf{p}_{2}\right)\right),
$$

and for any $\mathbf{p}$-triple $\left(\mathbf{q}_{1}^{\dagger}, \mathbf{q}_{2}^{\dagger}, \mathbf{q}_{3}^{\dagger}\right)$ with parameters $\left(\beta_{1}, \beta_{2}, \beta_{3}\right)$,

$$
\left[\mathbf{q}_{3}^{\dagger}\right]=\frac{\beta_{1}}{\beta_{1}+\beta_{2}}\left[\mathbf{q}_{1}^{\dagger}\right]\left(\mathbf{I}+\frac{\beta_{2}}{\beta_{1}} \mathbf{p}\left[\mathbf{q}_{2}^{\dagger}\right]\right)=\frac{1}{\beta_{1}+\beta_{2}} \frac{\partial}{\partial \mathbf{p}}\left(\beta_{1} \log \left(\mathbf{q}_{1}^{\dagger} \mathbf{p}\right)+\beta_{2} \log \left(\mathbf{q}_{2}^{\dagger} \mathbf{p}\right)\right) .
$$

\subsection{Gluing Properties}

The following two Lemmas show that any two triples with matching parts can be "glued" together and then completed into a cube in such a way that any vertex of the cube makes a triple. More precisely, the Face Lemma shows how to glue two triples of the same type that share two vertices, and the Edge Lemma shows how to glue two triples of different type if the center of one is the end of the other and vise-versa, and the common edge parameters coincide.

Lemma 3.3 (The Face Lemma). Let the pairs $\mathbf{p}_{1}, \mathbf{p}_{2} \in \mathbf{V}$ and $\mathbf{q}_{1}^{\dagger}, \mathbf{q}_{2}^{\dagger} \in \mathbf{V}^{\dagger}$ be linearly independent and not-orthogonal, $\mathbf{q}_{i}^{\dagger} \mathbf{p}_{j} \neq 0$, and let $\alpha_{1}, \alpha_{2}, \beta_{1}, \beta_{2}$ be any non-zero complex parameters. Then the quad 


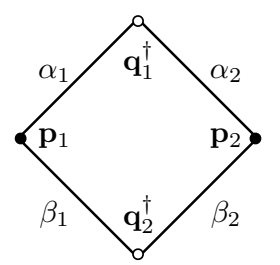

can be completed to a cube with

$$
\alpha_{3}=-\left(\alpha_{1}+\alpha_{2}\right), \quad \beta_{3}=-\left(\beta_{1}+\beta_{2}\right), \quad \gamma_{1}=-\left(\alpha_{1}+\beta_{1}\right), \quad \gamma_{2}=-\left(\alpha_{2}+\beta_{2}\right),
$$

and some other parameters $\alpha_{4}, \alpha_{5}, \beta_{4}, \beta_{5}$,
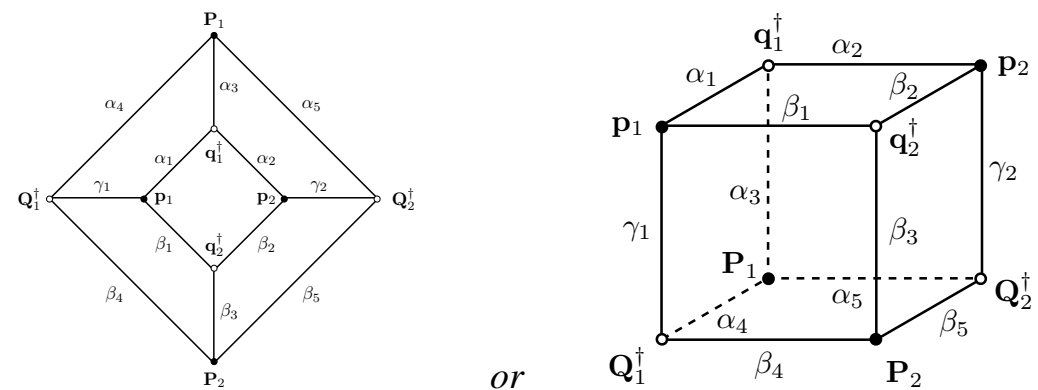

so that at each vertex the corresponding triple condition is satisfied. Moreover, if the parameters satisfy the additional cyclic condition

$$
\alpha_{1}+\alpha_{2}+\beta_{1}+\beta_{2}=0,
$$

on the initial face, then the same condition is satisfied on the remaining five faces, and so

$$
\alpha_{4}=-\beta_{2}, \quad \alpha_{5}=-\beta_{1}, \quad \beta_{4}=-\alpha_{2}, \quad \beta_{5}=-\alpha_{1} .
$$

Further, let

$$
\mathscr{L}=\alpha_{1} \log \left(\mathbf{q}_{1}^{\dagger} \mathbf{p}_{1}\right)+\alpha_{2} \log \left(\mathbf{q}_{1}^{\dagger} \mathbf{p}_{2}\right)+\beta_{1} \log \left(\mathbf{q}_{2}^{\dagger} \mathbf{p}_{1}\right)+\beta_{2} \log \left(\mathbf{q}_{1}^{\dagger} \mathbf{p}_{2}\right) .
$$

Then

$$
\left[\mathbf{P}_{1}\right]_{\mathbf{q}_{1}^{\dagger}}=\alpha_{3} \frac{\partial \mathscr{L}}{\partial \mathbf{q}_{1}^{\dagger}}, \quad\left[\mathbf{P}_{2}\right]_{\mathbf{q}_{2}^{\dagger}}=\beta_{3} \frac{\partial \mathscr{L}}{\partial \mathbf{q}_{2}^{\dagger}}, \quad\left[\mathbf{Q}_{1}^{\dagger}\right]_{\mathbf{p}_{1}}=\gamma_{1} \frac{\partial \mathscr{L}}{\partial \mathbf{p}_{1}}, \quad\left[\mathbf{Q}_{2}^{\dagger}\right]_{\mathbf{p}_{2}}=\gamma_{2} \frac{\partial \mathscr{L}}{\partial \mathbf{p}_{1}} .
$$

Proof. First, note that the normalized outside vertices are uniquely determined by the given data,

$$
\begin{array}{rlrl}
{\left[\mathbf{P}_{1}\right]_{\mathbf{q}_{1}^{\dagger}}=\frac{\alpha_{1}\left[\mathbf{p}_{1}\right]_{\mathbf{q}_{1}^{\dagger}}+\alpha_{2}\left[\mathbf{p}_{2}\right]_{\mathbf{q}_{1}^{\dagger}}}{\alpha_{1}+\alpha_{2}}} & {\left[\mathbf{P}_{2}\right]_{\mathbf{q}_{2}^{\dagger}}=\frac{\beta_{1}\left[\mathbf{p}_{1}\right]_{\mathbf{q}_{2}^{\dagger}}+\beta_{2}\left[\mathbf{p}_{2}\right]_{\mathbf{q}_{2}^{\dagger}}}{\beta_{1}+\beta_{2}}} \\
{\left[\mathbf{Q}_{1}^{\dagger}\right]_{\mathbf{p}_{1}}=\frac{\alpha_{1}\left[\mathbf{q}_{1}^{\dagger}\right]_{\mathbf{p}_{1}+\beta_{1}\left[\mathbf{q}_{2}^{\dagger}\right] \mathbf{p}_{1}}^{\alpha_{1}+\beta_{1}}}{\left[\mathbf{Q}_{2}^{\dagger}\right]_{\mathbf{p}_{2}}}=\frac{\alpha_{2}\left[\mathbf{q}_{1}^{\dagger}\right] \mathbf{p}_{2}+\beta_{2}\left[\mathbf{q}_{2}^{\dagger}\right]_{\mathbf{p}_{2}}}{\alpha_{2}+\beta_{2}} .}
\end{array}
$$

Thus, to prove the theorem we need to show that it is possible to find the parameters $\alpha_{4}, \alpha_{5}, \beta_{4}, \beta_{5}$ so that

$$
\alpha_{4}+\alpha_{5}=\alpha_{1}+\alpha_{2}, \quad \beta_{4}+\beta_{5}=\beta_{1}+\beta_{2}, \quad \alpha_{4}+\beta_{4}=\alpha_{1}+\beta_{1}, \quad \alpha_{5}+\beta_{5}=\alpha_{2}+\beta_{2},
$$


and that the following equations hold at the outside nodes:

$$
\begin{aligned}
& \mathbf{Q}_{1}^{\dagger}: \quad \alpha_{4}\left[\mathbf{P}_{1}\right]_{\mathbf{Q}_{1}^{\dagger}}+\beta_{4}\left[\mathbf{P}_{2}\right]_{\mathbf{Q}_{1}^{\dagger}}+\gamma_{1}\left[\mathbf{p}_{1}\right]_{\mathbf{Q}_{1}^{\dagger}}=\mathbf{0} \\
& \mathbf{Q}_{2}^{\dagger}: \quad \alpha_{5}\left[\mathbf{P}_{1}\right]_{\mathbf{Q}_{2}^{\dagger}}+\beta_{5}\left[\mathbf{P}_{2}\right]_{\mathbf{Q}_{2}^{\dagger}}+\gamma_{2}\left[\mathbf{p}_{2}\right]_{\mathbf{Q}_{2}^{\dagger}}=\mathbf{0} \\
& \mathbf{P}_{1}: \quad \alpha_{4}\left[\mathbf{Q}_{1}^{\dagger}\right] \mathbf{P}_{1}+\alpha_{5}\left[\mathbf{Q}_{2}^{\dagger}\right] \mathbf{P}_{1}+\alpha_{3}\left[\mathbf{q}_{1}^{\dagger}\right] \mathbf{P}_{1}=\mathbf{0} \\
& \mathbf{P}_{2}: \quad \beta_{4}\left[\mathbf{Q}_{1}^{\dagger}\right] \mathbf{P}_{2}+\beta_{5}\left[\mathbf{Q}_{2}^{\dagger}\right] \mathbf{P}_{2}+\beta_{3}\left[\mathbf{q}_{2}^{\dagger}\right] \mathbf{P}_{2}=\mathbf{0} .
\end{aligned}
$$

Using the change of normalization rule

$$
[\mathbf{p}]_{\mathbf{q}_{i}}=\frac{\mathbf{q}_{j}^{\dagger} \mathbf{p}}{\mathbf{q}_{i}^{\dagger} \mathbf{p}}[\mathbf{p}]_{\mathbf{q}_{j}^{\dagger}}
$$

the first equation, when decomposed in the spanning basis $\mathbf{p}_{1}, \mathbf{p}_{2}$, becomes the following system:

$$
\left\{\begin{array}{c}
\left(\frac{\alpha_{4}}{\alpha_{1}+\alpha_{2}} \frac{\mathbf{q}_{1}^{\dagger} \mathbf{P}_{1}}{\mathbf{Q}_{1}^{\dagger} \mathbf{P}_{1}}\right) \cdot \alpha_{1} \frac{\mathbf{Q}_{1}^{\dagger} \mathbf{p}_{1}}{\mathbf{q}_{1}^{\dagger} \mathbf{p}_{1}}+\left(\frac{\beta_{4}}{\beta_{1}+\beta_{2}} \frac{\mathbf{q}_{2}^{\dagger} \mathbf{P}_{2}}{\mathbf{Q}_{1}^{\dagger} \mathbf{P}_{2}}\right) \cdot \beta_{1} \frac{\mathbf{Q}_{1}^{\dagger} \mathbf{p}_{1}}{\mathbf{q}_{2}^{\dagger} \mathbf{p}_{1}}=\alpha_{1}+\beta_{1} \\
\left(\frac{\alpha_{4}}{\alpha_{1}+\alpha_{2}} \frac{\mathbf{q}_{1}^{\dagger} \mathbf{P}_{1}}{\mathbf{Q}_{1}^{\dagger} \mathbf{P}_{1}}\right) \cdot \alpha_{2} \frac{\mathbf{q}_{2}^{\dagger} \mathbf{p}_{2}}{\mathbf{q}_{1}^{\dagger} \mathbf{p}_{2}}+\left(\frac{\beta_{4}}{\beta_{1}+\beta_{2}} \frac{\mathbf{q}_{2}^{\dagger} \mathbf{P}_{2}}{\mathbf{Q}_{1}^{\dagger} \mathbf{P}_{2}}\right) \cdot \beta_{2}=0
\end{array}\right.
$$

Solving it gives

$$
\begin{aligned}
& \alpha_{4}=\beta_{2}\left(\alpha_{1}+\alpha_{2}\right)\left(\alpha_{1}+\beta_{1}\right)\left[\mathbf{Q}_{1}^{\dagger}\right]_{\mathbf{p}_{1}}\left[\mathbf{P}_{1}\right]_{\mathbf{q}_{1}^{\dagger}} \frac{\mathbf{q}_{1}^{\dagger} \mathbf{p}_{1} \mathbf{q}_{2}^{\dagger} \mathbf{p}_{1} \mathbf{q}_{1}^{\dagger} \mathbf{p}_{2}}{\alpha_{1} \beta_{2} \mathbf{q}_{2}^{\dagger} \mathbf{p}_{1} \mathbf{q}_{1}^{\dagger} \mathbf{p}_{2}-\alpha_{2} \beta_{1} \mathbf{q}_{1}^{\dagger} \mathbf{p}_{1} \mathbf{q}_{2}^{\dagger} \mathbf{p}_{2}}, \\
& \beta_{4}=\alpha_{2}\left(\beta_{1}+\beta_{2}\right)\left(\alpha_{1}+\beta_{1}\right)\left[\mathbf{Q}_{1}^{\dagger}\right]_{\mathbf{p}_{1}}\left[\mathbf{P}_{2}\right]_{\mathbf{q}_{2}^{\dagger}} \frac{-\mathbf{q}_{1}^{\dagger} \mathbf{p}_{1} \mathbf{q}_{2}^{\dagger} \mathbf{p}_{1} \mathbf{q}_{1}^{\dagger} \mathbf{p}_{2}}{\alpha_{1} \beta_{2} \mathbf{q}_{2}^{\dagger} \mathbf{p}_{1} \mathbf{q}_{1}^{\dagger} \mathbf{p}_{2}-\alpha_{2} \beta_{1} \mathbf{q}_{1}^{\dagger} \mathbf{p}_{1} \mathbf{q}_{2}^{\dagger} \mathbf{p}_{2}},
\end{aligned}
$$

which, together with the expressions

$$
\begin{aligned}
& {\left[\mathbf{Q}_{1}^{\dagger}\right]_{\mathbf{p}_{1}}\left[\mathbf{P}_{1}\right]_{\mathbf{q}_{1}^{\dagger}}=\frac{-1}{\left(\alpha_{1}+\alpha_{2}\right)\left(\alpha_{1}+\beta_{1}\right)} \cdot \frac{\alpha_{1}\left(\beta_{2}-\kappa\right) \mathbf{q}_{2}^{\dagger} \mathbf{p}_{1} \mathbf{q}_{1}^{\dagger} \mathbf{p}_{2}-\alpha_{2} \beta_{1} \mathbf{q}_{1}^{\dagger} \mathbf{p}_{1} \mathbf{q}_{2}^{\dagger} \mathbf{p}_{2}}{\mathbf{q}_{1}^{\dagger} \mathbf{p}_{1} \mathbf{q}_{2}^{\dagger} \mathbf{p}_{1} \mathbf{q}_{1}^{\dagger} \mathbf{p}_{2}}} \\
& {\left[\mathbf{Q}_{1}^{\dagger}\right]_{\mathbf{p}_{1}}\left[\mathbf{p}_{2}\right]_{\mathbf{q}_{2}^{\dagger}}=\frac{1}{\left(\beta_{1}+\beta_{2}\right)\left(\alpha_{1}+\beta_{1}\right)} \cdot \frac{\alpha_{1} \beta_{2} \mathbf{q}_{2}^{\dagger} \mathbf{p}_{1} \mathbf{q}_{1}^{\dagger} \mathbf{p}_{2}-\left(\alpha_{2}-\kappa\right) \beta_{1} \mathbf{q}_{1}^{\dagger} \mathbf{p}_{1} \mathbf{q}_{2}^{\dagger} \mathbf{p}_{2}}{\mathbf{q}_{1}^{\dagger} \mathbf{p}_{1} \mathbf{q}_{2}^{\dagger} \mathbf{p}_{1} \mathbf{q}_{2}^{\dagger} \mathbf{p}_{2}},}
\end{aligned}
$$

where $\kappa=\alpha_{1}+\alpha_{2}+\beta_{1}+\beta_{2}$, gives

$$
\begin{aligned}
& \alpha_{4}=-\beta_{2}+\kappa \frac{\alpha_{1} \beta_{2} \mathbf{q}_{2}^{\dagger} \mathbf{p}_{1} \mathbf{q}_{1}^{\dagger} \mathbf{p}_{2}}{\alpha_{1} \beta_{2} \mathbf{q}_{2}^{\dagger} \mathbf{p}_{1} \mathbf{q}_{1}^{\dagger} \mathbf{p}_{2}-\alpha_{2} \beta_{1} \mathbf{q}_{1}^{\dagger} \mathbf{p}_{1} \mathbf{q}_{2}^{\dagger} \mathbf{p}_{2}}, \\
& \beta_{4}=-\alpha_{2}+\kappa \frac{-\alpha_{2} \beta_{1} \mathbf{q}_{1}^{\dagger} \mathbf{p}_{1} \mathbf{q}_{2}^{\dagger} \mathbf{p}_{2}}{\alpha_{1} \beta_{2} \mathbf{q}_{2}^{\dagger} \mathbf{p}_{1} \mathbf{q}_{1}^{\dagger} \mathbf{p}_{2}-\alpha_{2} \beta_{1} \mathbf{q}_{1}^{\dagger} \mathbf{p}_{1} \mathbf{q}_{2}^{\dagger} \mathbf{p}_{2}},
\end{aligned}
$$

and so it is easy to see that $\alpha_{4}+\beta_{4}=\kappa-\alpha_{2}-\beta_{2}=\alpha_{1}+\beta_{1}$, as required. Similarly, using

$$
\begin{aligned}
& {\left[\mathbf{Q}_{2}^{\dagger}\right]_{\mathbf{p}_{2}}\left[\mathbf{P}_{1}\right]_{\mathbf{q}_{1}^{\dagger}}=\frac{1}{\left(\alpha_{1}+\alpha_{2}\right)\left(\alpha_{2}+\beta_{2}\right)} \cdot \frac{\alpha_{1} \beta_{2} \mathbf{q}_{2}^{\dagger} \mathbf{p}_{1} \mathbf{q}_{1}^{\dagger} \mathbf{p}_{2}-\alpha_{2}\left(\beta_{1}-\kappa\right) \mathbf{q}_{1}^{\dagger} \mathbf{p}_{1} \mathbf{q}_{2}^{\dagger} \mathbf{p}_{2}}{\mathbf{q}_{1}^{\dagger} \mathbf{p}_{1} \mathbf{q}_{1}^{\dagger} \mathbf{p}_{2} \mathbf{q}_{2}^{\dagger} \mathbf{p}_{2}}} \\
& {\left[\mathbf{Q}_{2}^{\dagger}\right]_{\mathbf{p}_{2}}\left[\mathbf{P}_{2}\right]_{\mathbf{q}_{2}^{\dagger}}=\frac{-1}{\left(\beta_{1}+\beta_{2}\right)\left(\alpha_{2}+\beta_{2}\right)} \cdot \frac{\left(\alpha_{1}-\kappa\right) \beta_{2} \mathbf{q}_{2}^{\dagger} \mathbf{p}_{1} \mathbf{q}_{1}^{\dagger} \mathbf{p}_{2}-\alpha_{2} \beta_{1} \mathbf{q}_{1}^{\dagger} \mathbf{p}_{1} \mathbf{q}_{2}^{\dagger} \mathbf{p}_{2}}{\mathbf{q}_{2}^{\dagger} \mathbf{p}_{1} \mathbf{q}_{1}^{\dagger} \mathbf{p}_{2} \mathbf{q}_{2}^{\dagger} \mathbf{p}_{2}},}
\end{aligned}
$$


and the equations at the node $\mathbf{Q}_{2}^{\dagger}$ gives

$$
\begin{aligned}
& \alpha_{5}=-\beta_{1}+\kappa \frac{-\alpha_{2} \beta_{1} \mathbf{q}_{1}^{\dagger} \mathbf{p}_{1} \mathbf{q}_{2}^{\dagger} \mathbf{p}_{2}}{\alpha_{1} \beta_{2} \mathbf{q}_{2}^{\dagger} \mathbf{p}_{1} \mathbf{q}_{1}^{\dagger} \mathbf{p}_{2}-\alpha_{2} \beta_{1} \mathbf{q}_{1}^{\dagger} \mathbf{p}_{1} \mathbf{q}_{2}^{\dagger} \mathbf{p}_{2}}, \\
& \beta_{5}=-\alpha_{1}+\kappa \frac{\alpha_{1} \beta_{2} \mathbf{q}_{2}^{\dagger} \mathbf{p}_{1} \mathbf{q}_{1}^{\dagger} \mathbf{p}_{2}}{\alpha_{1} \beta_{2} \mathbf{q}_{2}^{\dagger} \mathbf{p}_{1} \mathbf{q}_{1}^{\dagger} \mathbf{p}_{2}-\alpha_{2} \beta_{1} \mathbf{q}_{1}^{\dagger} \mathbf{p}_{1} \mathbf{q}_{2}^{\dagger} \mathbf{p}_{2}} .
\end{aligned}
$$

Similar computations show that equations at the nodes $\mathbf{P}_{1}$ and $\mathbf{P}_{2}$ give the same values of the parameters, and the other constraints are easy to check.

Lemma 3.4 (The Edge Lemma). Let $\left(\mathbf{p}_{1}, \mathbf{p}_{2}, \mathbf{p}_{3}\right)$ be a $\mathbf{q}_{3}^{\dagger}$-triple with parameters $\left(\alpha_{1}, \alpha_{2}, \alpha_{3}\right)$, $\left(\mathbf{q}_{1}^{\dagger}, \mathbf{q}_{2}^{\dagger}, \mathbf{q}_{3}^{\dagger}\right)$ be a $\mathbf{p}_{3}$-triple with parameters $\left(\beta_{1}, \beta_{2}, \beta_{3}\right)$ with parameters $\left(\beta_{1}, \beta_{2}, \beta_{3}\right)$, and let $\alpha_{3}=\beta_{3}$,
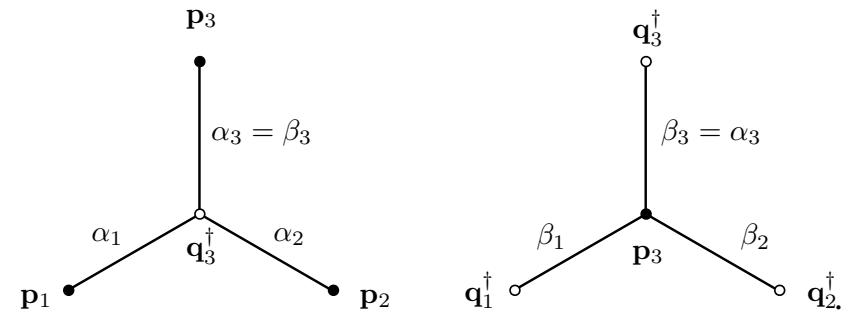

Then it is possible to glue the triples along the $\mathbf{p}_{3} \mathbf{q}_{3}^{\dagger}$-edge and, by assigning parameters $\gamma_{1}$ and $\gamma_{2}$ along the $\mathbf{p}_{1} \mathbf{q}_{1}^{\dagger}$ and $\mathbf{p}_{2} \mathbf{q}_{2}^{\dagger}$ edges so that the sum of parameters in the new quads is zero (i.e., $\gamma_{1}=\alpha_{2}-\beta_{1}$ and $\gamma_{2}=\alpha_{1}-\beta_{2}$ ), complete the resulting "butterfly" configuration to a consistent cube,
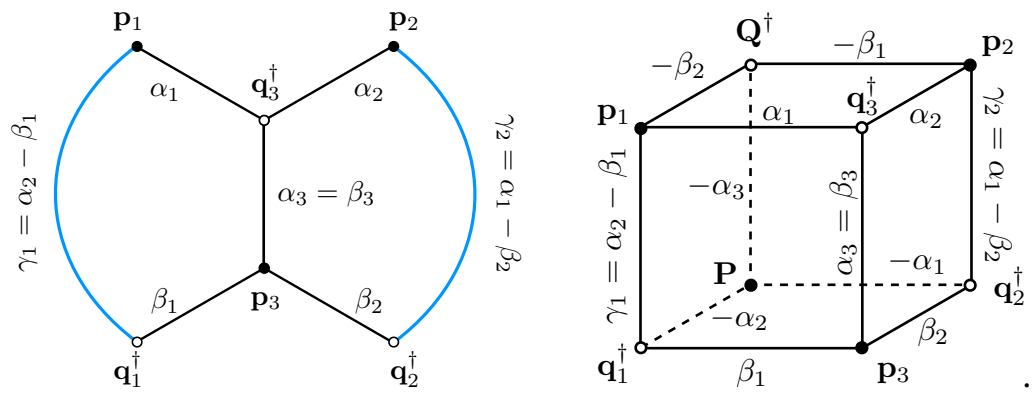

Proof. After choosing $\gamma_{1}$ and $\gamma_{2}$ according to the above rule, this follows immediately from the Face Lemma 3.3.

\section{Eigenvectors of Quadratic Lax Matrices and Refactorization}

In this section we show that a cube diagram described in the previous section can be used to represent the relationship between eigenvectors of quadratic Lax matrices. Also, we show that the three axes of symmetry of the cube connecting the centers of opposing sides can be given the following interpretation. One of the axes corresponds to switching the coordinate system on the space from left to right divisors. The other two axes correspond to two different directions of the refactorization dynamics corresponding to two possible pairings between two zeroes and two poles of $\operatorname{det} \mathbf{L}(z)$. For each of this transformations we produce the explicit formula for the generating function using the labeling of the cube. 


\subsection{Relations Between Eigenvectors of Quadratic Lax Matrices}

Consider a quadratic Lax matrix $\mathbf{L}(z)$ given in the additive form as

$$
\begin{aligned}
\mathbf{L}(z) & =\mathbf{L}_{0}+\frac{\mathbf{a}_{1} \mathbf{b}_{1}^{\dagger}}{z-z_{1}}+\frac{\mathbf{a}_{2} \mathbf{b}_{2}^{\dagger}}{z-z_{2}}, \quad \mathbf{L}(z)^{-1}=\mathbf{L}_{0}^{-1}-\frac{\mathbf{c}_{\alpha} \mathbf{d}_{\alpha}^{\dagger}}{z-\zeta_{\alpha}}-\frac{\mathbf{c}_{\beta} \mathbf{d}_{\beta}^{\dagger}}{z-\zeta_{\beta}}, \\
\operatorname{det} \mathbf{L}(z) & =\operatorname{det} \mathbf{L}_{0} \frac{\left(z-\zeta_{\alpha}\right)\left(z-\zeta_{\beta}\right)}{\left(z-z_{1}\right)\left(z-z_{2}\right)} .
\end{aligned}
$$

Pairing $z_{1}$ with $\zeta_{\alpha}=\zeta_{1}$ and $z_{2}$ with $\zeta_{\beta}=\zeta_{2}$ we can write $\mathbf{L}(z)$ and $\mathbf{L}^{-1}(z)$ in the multiplicative form using factors $\mathbf{B}_{i}(z)=\mathbf{I}+\frac{z-\zeta_{i}}{z-z_{i}} \frac{\mathbf{p}_{i} \mathbf{q}_{i}^{\dagger}}{\mathbf{q}_{i}^{\dagger} \mathbf{p}_{i}}$,

$$
\mathbf{L}(z)=\mathbf{L}_{0} \mathbf{B}_{1}(z) \mathbf{B}_{2}(z), \quad \mathbf{L}(z)^{-1}=\mathbf{B}_{2}(z)^{-1} \mathbf{B}_{1}(z)^{-1} \mathbf{L}_{0}^{-1},
$$

and so $\mathbf{B}_{1}(z)=\mathbf{L}_{0}^{-1} \mathbf{B}_{1}^{l}(z) \mathbf{L}_{0}=\mathbf{I}+\frac{z_{1}-\zeta_{\alpha}}{z-z_{1}} \frac{\mathbf{L}_{0}^{-1} \mathbf{a}_{1} \mathbf{d}_{\alpha}^{\dagger} \mathbf{L}_{0}}{\mathbf{d}_{\alpha}^{\dagger} \mathbf{a}_{1}}$ and $\mathbf{B}_{2}(z)=\mathbf{B}_{2}^{r}(z)=\mathbf{I}+\frac{z_{2}-\zeta_{\beta}}{z-z_{2}} \frac{\mathbf{c}_{\beta} \mathbf{b}_{2}^{\dagger}}{\mathbf{b}_{2}^{\dagger} \mathbf{c}_{\beta}}$. This can also be seen directly by looking at the residues of $\mathbf{L}(z)$ at $z_{1}$ and $z_{2}$ and of $\mathbf{L}(z)^{-1}$ at $\zeta_{\alpha}$ and $\zeta_{\beta}$; in fact, that is how the expressions for the right and left divisors are obtained. The same residues also give the following collection of equations:

$$
\mathbf{d}_{\alpha}^{\dagger} \mathbf{L}_{0} \mathbf{B}_{2}\left(z_{1}\right) \sim \mathbf{b}_{1}^{\dagger}, \quad \mathbf{B}_{1}\left(z_{2}\right) \mathbf{c}_{\beta} \sim \mathbf{L}_{0}^{-1} \mathbf{a}_{2}, \quad \mathbf{B}_{2}\left(\zeta_{\alpha}\right) \mathbf{c}_{\alpha} \sim \mathbf{L}_{0}^{-1} \mathbf{a}_{1}, \quad \mathbf{d}_{\beta}^{\dagger} \mathbf{L}_{0} \mathbf{B}_{1}\left(\zeta_{\beta}\right) \sim \mathbf{b}_{2}^{\dagger} .
$$

Representing these equations using elementary triples and gluing, we get the following result.

Theorem 4.1. The eigenvectors of $\mathbf{L}(z)$ are related by the following cube diagram:

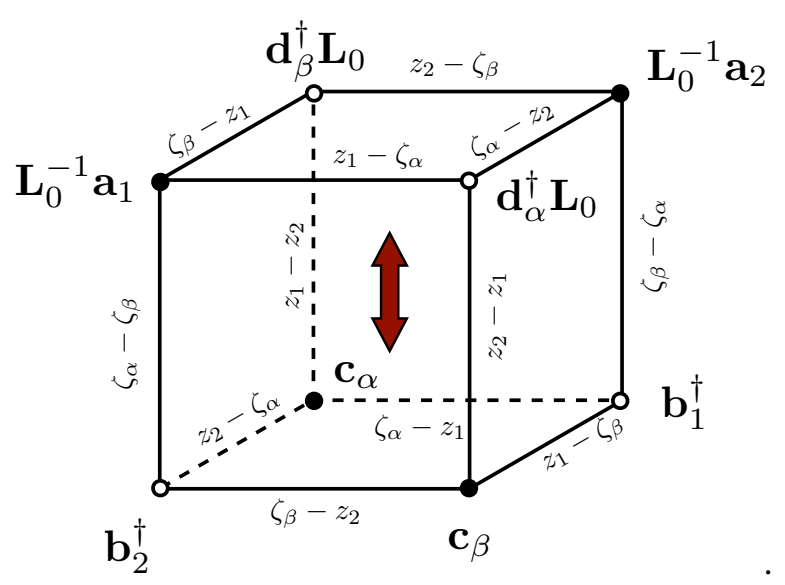

Note that in this diagram, the top (resp. bottom) faces of the cube correspond to parameterizing the space of quadratic Lax matrices by left (resp. right) divisors, and so the vertical axis of the cube correspond to the change of coordinates between these two different systems. Two horizontal axes correspond to the refactorization dynamics.

\subsection{Refactorization Dynamics}

Consider now an isospectral dynamic $\mathbf{L}(z) \mapsto \tilde{\mathbf{L}}(z)=\mathbf{R}(z) \mathbf{L}(z) \mathbf{R}(z)^{-1}$, the isomonodromic case is similar. If we take $\mathbf{R}(z)=\mathbf{B}_{2}(z)=\mathbf{B}_{\beta ; 2}^{r}(z)$, where the notation $\mathbf{B}_{\beta ; 2}^{r}(z)$ explicitly specifies the zero 
and the pole of the elementary divisor, this becomes a refactorization transformation

$$
\mathbf{L}(z)=\mathbf{L}_{0} \mathbf{B}_{\alpha ; 1}(z) \mathbf{B}_{\beta ; 2}(z) \mapsto \tilde{\mathbf{L}}(z)=\mathbf{B}_{\beta ; 2}(z) \mathbf{L}_{0} \mathbf{B}_{\alpha ; 1}(z)=\mathbf{L}_{0} \tilde{\mathbf{B}}_{\alpha ; 1}(z) \tilde{\mathbf{B}}_{\beta ; 2}(z) .
$$

Since we can think of the refactorization transformation as switching the roles of the left and right divisors, $\mathbf{B}_{\beta, 2}(z)=\mathbf{B}_{\beta, 2}^{r}(z)=\tilde{\mathbf{B}}_{\beta, 2}^{l}(z)$ and $\mathbf{B}_{\alpha, 1}(z)=\mathbf{L}_{0}^{-1} \mathbf{B}_{\alpha, 1}^{l}(z) \mathbf{L}_{0}=\tilde{\mathbf{B}}_{\alpha, 1}^{r}(z)$, using (2.10) we get the identifications $\tilde{\mathbf{a}}_{2}=\mathbf{c}_{\beta}, \tilde{\mathbf{d}}_{\beta}^{\dagger}=\mathbf{b}_{2}, \tilde{\mathbf{c}}_{\alpha}=\mathbf{L}_{0}^{-1} \mathbf{a}_{1}$, and $\tilde{\mathbf{b}}_{1}^{\dagger}=\mathbf{d}_{\alpha}^{\dagger} \mathbf{L}_{0}$. Thus, on the diagram below labels on the back face correspond to the coordinates of $\mathbf{L}(z)$, labels on the front face correspond (after twisting $\tilde{\mathbf{a}}_{2}$ by $\mathbf{L}_{0}^{-1}$ and $\tilde{\mathbf{d}}_{\beta}^{\dagger}$ by $\left.\mathbf{L}_{0}\right)$ to the coordinates of $\tilde{\mathbf{L}}(z)$,

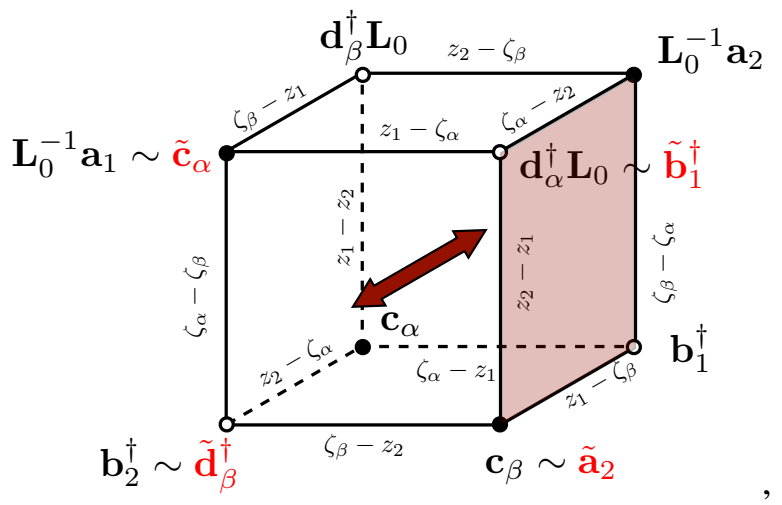

and the generating function encoded by the right face of the cube is, up to some minor change of notation, exactly the Lagrangian from Theorem (2.1),

$$
\begin{aligned}
\left.\mathscr{L}\left(\left(\mathbf{a}_{2}, \mathbf{b}_{1}^{\dagger}\right), \widetilde{\left(\mathbf{a}_{2}, \mathbf{b}_{1}^{\dagger}\right.}\right)\right)= & \left(z_{2}-z_{1}\right) \log \left(\tilde{\mathbf{b}}_{1}^{\dagger} \tilde{\mathbf{a}}_{2}\right)+\left(z_{1}-\zeta_{\beta}\right) \log \left(\mathbf{b}_{1}^{\dagger} \tilde{\mathbf{a}}_{2}\right) \\
& +\left(\zeta_{\beta}-\zeta_{\alpha}\right) \log \left(\mathbf{b}_{1}^{\dagger} \mathbf{L}_{0}^{-1} \mathbf{a}_{2}\right)+\left(\zeta_{\alpha}-z_{2}\right) \log \left(\tilde{\mathbf{b}}_{1}^{\dagger} \mathbf{L}_{0}^{-1} \mathbf{a}_{2}\right) .
\end{aligned}
$$

Note that using the generating function given by the left face of the cube corresponds to the backwards motion generated by $\mathbf{R}(z)=\mathbf{B}_{\alpha ; 1}^{r}(z)$.

The remaining axis of the cube corresponds to pairing $z_{1}$ with $\zeta_{\beta}$ and $z_{2}$ with $\zeta_{\alpha}$. In other words, taking $\mathbf{R}(z)=\mathbf{B}_{\alpha ; 2}^{r}(z)$ (or $\mathbf{R}(z)=\mathbf{B}_{\beta ; 1}^{r}(z)$ for the backwards motion),

$$
\mathbf{L}(z)=\mathbf{L}_{0} \mathbf{B}_{\beta ; 1}(z) \mathbf{B}_{\alpha ; 2}(z) \mapsto \tilde{\mathbf{L}}(z)=\mathbf{B}_{\alpha ; 2}(z) \mathbf{L}_{0} \mathbf{B}_{\beta ; 1}(z)=\mathbf{L}_{0} \tilde{\mathbf{B}}_{\beta ; 1}(z) \tilde{\mathbf{B}}_{\alpha ; 2}(z),
$$

we get the following map

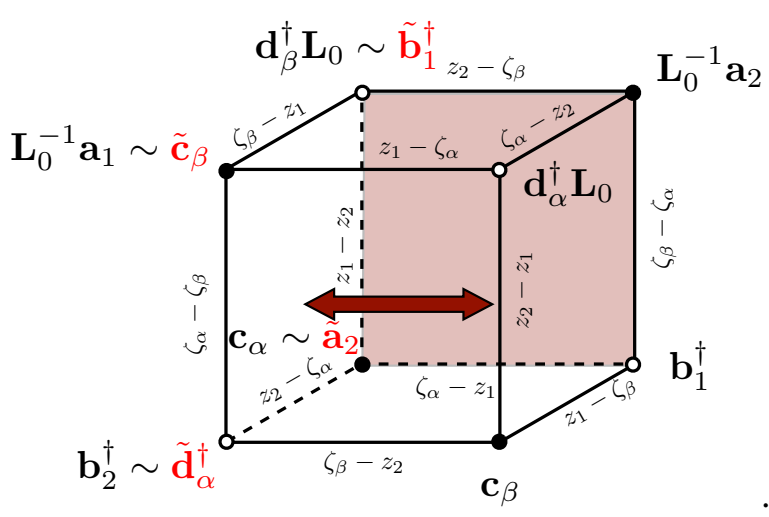


and its Lagrangian generating function

$$
\begin{aligned}
\left.\mathscr{L}\left(\left(\mathbf{a}_{2}, \mathbf{b}_{1}^{\dagger}\right), \widetilde{\left(\mathbf{a}_{2}, \mathbf{b}_{1}^{\dagger}\right.}\right)\right)= & \left(z_{1}-z_{2}\right) \log \left(\tilde{\mathbf{b}}_{1}^{\dagger} \tilde{\mathbf{a}}_{2}\right)+\left(\zeta_{\alpha}-z_{1}\right) \log \left(\mathbf{b}_{1}^{\dagger} \tilde{\mathbf{a}}_{2}\right) \\
& +\left(\zeta_{\beta}-\zeta_{\alpha}\right) \log \left(\mathbf{b}_{1}^{\dagger} \mathbf{L}_{0}^{-1} \mathbf{a}_{2}\right)+\left(z_{2}-\zeta_{\beta}\right) \log \left(\tilde{\mathbf{b}}_{1}^{\dagger} \mathbf{L}_{0}^{-1} \mathbf{a}_{2}\right) .
\end{aligned}
$$

\section{Conclusions}

We explained a neat and efficient way to encode the structure of refactorization transformations for quadratic Lax matrices and their generating functions using cube diagrams. This approach also gives different ways of choosing coordinate systems on the space of such matrices - each choice corresponds to a face of the cube. It would be very interesting to see if this approach can be generalized to Lax matrices with more than two factors. Since, in view of the gluing properties, cube diagrams are rigid, we expect it to results in higher dimensional configurations relating such cubes, where each cube represents a particular transposition of factors. In particular, the case of three factors is related to the structure of Yang-Baxter maps and we plan to consider it in a separate publication.

\section{Acknowledgements}

The author thanks Adam Doliwa, Michael Gekhtman, and Yuri Suris for helpful conversations and suggestions.

\section{References}

[1] Alexei Borodin, Isomonodromy transformations of linear systems of difference equations, Ann. of Math. (2) 160 (2004), no. 3, 1141-1182. MR MR2144976

[2] Alexander I. Bobenko and Yuri B. Suris, Integrable systems on quad-graphs, Int. Math. Res. Not. (2002), no. 11, 573-611. MR MR1890049 (2003d:37127)

[3] Anton Dzhamay, On the Lagrangian structure of the discrete isospectral and isomonodromic transformations, Int. Math. Res. Not. IMRN (2008), Art. ID rnn 102, 22. MR MR2439540

[4] - Factorizations of rational matrix functions with application to discrete isomonodromic transformations and difference Painlevé equations, J. Phys. A: Math. Theor. 42 (2009), no. 45, 10pp.

[5] V. M. Goncharenko and A. P. Veselov, Yang-Baxter maps and matrix solitons, New trends in integrability and partial solvability, NATO Sci. Ser. II Math. Phys. Chem., vol. 132, Kluwer Acad. Publ., Dordrecht, 2004, pp. 191-197. MR 2153338 (2006c:81074)

[6] I. M. Krichever and D. H. Phong, Symplectic forms in the theory of solitons, Surveys in differential geometry: integral systems [integrable systems], Surv. Differ. Geom., IV, Int. Press, Boston, MA, 1998, pp. 239-313. MR 1726930 (2001k:37114)

[7] Sarah Lobb and Frank Nijhoff, Lagrangian multiforms and multidimensional consistency, J. Phys. A 42 (2009), no. 45, 454013, 18. MR MR2556655

[8] Jürgen Moser and Alexander P. Veselov, Discrete versions of some classical integrable systems and factorization of matrix polynomials, Comm. Math. Phys. 139 (1991), no. 2, 217-243. MR MR1120138 (92g:58054)

[9] F. W. Nijhoff, Lax pair for the Adler (lattice Krichever-Novikov) system, Phys. Lett. A 297 (2002), no. 1-2, 49-58. MR 1912127 (2003f:37121)

[10] Barbara Prinari, Mark J. Ablowitz, and Gino Biondini, Inverse scattering transform for the vector nonlinear Schrödinger equation with nonvanishing boundary conditions, J. Math. Phys. 47 (2006), no. 6, 063508, 33. MR 2239983 (2007f:37118)

[11] Hidetaka Sakai, Rational surfaces associated with affine root systems and geometry of the Painlevé equations, Comm. Math. Phys. 220 (2001), no. 1, 165-229. MR MR1882403 (2003c:14030) 
Anton Dzhamay

[12] A. P. Veselov, Integrable systems with discrete time, and difference operators, Funktsional. Anal. i Prilozhen. 22 (1988), no. 2, 1-13, 96. MR MR947601 (90a:58081)

[13] Integrable Lagrangian correspondences and factorization of matrix polynomials, Funktsional. Anal. i Prilozhen. 25 (1991), no. 2, 38-49, 96. MR MR1142207 (93b:58081)

[14] V. E. Zaharov and A. V. Mihallov, A variational principle for equations that are integrable by the inverse problem method, Funktsional. Anal. i Prilozhen. 14 (1980), no. 1, 55-56. MR MR565100 (81c:35111) 